\title{
COMPARISON OF SULA LIQUID MEDIA AND CONVENTIONAL METHOD FOR DIAGNOSIS OF MDR-TB
}

\author{
IQBAL $\mathrm{H}^{1}$, MAMUN KZ2 ${ }^{2}$, SHAMSUZZAMAN SM${ }^{3}$, KAMAL SMM ${ }^{4}$
}

\begin{abstract}
:
Background: Tuberculosis is probably the most important infectious disease of human. Early diagnosis of tuberculosis and initiating prompt treatment has been the mainstay in interrupting the transmission of the infection to others in the community.
\end{abstract}

Aim: The present study was aimed at evaluating and comparing the different media which are available for the diagnosis of tuberculosis and at determining their feasibility as diagnostic tests in terms of their effectiveness and affordability.

Methods: $100 \mathrm{Z}-\mathrm{N}$ stain positive samples were taken from NIDCH, Mohakhali, Dhaka. All samples were cultured on LJ media for 4-6 wks and sula liquid media for 12 days. Drug sensitivity tests against isoniazid (INH), rifampicin (RIF), streptomycin (SM) and ethambutol $(E M B)$ were done by both conventional ( $L J$ media) and slide method and additional sensitivity were done against second line drugs such as gatifloxacin (G), kanamycin (K) and ofloxacin (OF) only by slide method. Sensitivity test against P-nitro benzoic acid (PNB) were also done to differentiate typical Mycobacterium from atypical Mycobacterium by both slide and conventional methods.

Results: Of the 100 sputum samples which were inoculated onto the sula liquid and LJ medium, all showed growth of Mycobacterium tuberculosis on sula liquid media after a mean incubation period of 7 days and on $L J$ medium after a mean incubation period of 34 days.

Conclusion: Sula liquid media had an advantage of a shorter turn around period of 12 days when compared with conventional L-J media.

J Dhaka Med Coll. 2016; 25(2) : 100-104

\section{Introduction}

Tuberculosis (TB), for many centuries, has been the most important of human infections, in its global prevalence, devastating morbidity and massive mortality. It has been estimated that a third of the world's population is infected with the tubercle bacilli. Each year, more than eight million new cases and three million deaths due to tuberculosis is being reported worldwide ${ }^{1}$.The prevalence of multi-drug resistant tuberculosis (MDR-TB) and extensively-drug resistant tuberculosis (XDRTB) are increasing throughout the world both among new tuberculosis cases as well as among previously treated ones ${ }^{2}$. MDR-TB is a form of TB caused by a strain of $M$. tuberculosis resistant to the most potent first line anti- tuberculous drugs, i.e. isoniazid (INH) and rifampicin (RIF). It has been estimated that India and China account for nearly $50 \%$ of the global burden of MDR-TB cases ${ }^{3}$. However, during the last decade, there has been an increase in reported incidences of drug resistance in Category II cases, particularly among those treated irregularly, or with incorrect regimens and doses. In such cases, the incidence of MDR-TB varies from $12-17 \%{ }^{4}$.

XDR-TB is defined as TB caused by a strain of M. tuberculosis that is resistant to rifampicin and isoniazid, as well as to any member of the quinolone family and at least one of the secondline anti-tuberculous injectable drugs i.e., Kanamycin, Capreomycin, or Amikacin. XDR-

1. Dr. Hasina Iqbal, Assistant Professor, Dept. of Microbiology, Popular Medical College, Dhaka.

2. Professor K. Z. Mamun, Head, Dept. of Microbiology, Popular Medical College, Dhaka.

3. Professor S. M. Shamsuzzaman, Dept. of Microbiology, Dhaka Medical College, Dhaka.

4. Dr. S. M. Mostafa Kamal Assoc Professor, Dept. of Microbiology, NIDCH, Mohakhali, Dhaka.

Correspondence: Dr. Hasina Iqbal, Assistant Professor, Dept. of Microbiology, Popular Medical College, Dhaka. E- mail: dr.hasinaiqbal@yahoo.com.

Received: 07 July 2016

Accepted: 01 September 2016 
TB was first described in 2006. Since then, there have been documented cases in six continents and 55 countries ${ }^{5}$. The global prevalence of XDR-TB has been difficult to assess ${ }^{6}$. Treatment outcomes are significantly worse for patients with XDR-TB, compared to patients with drug-susceptible TB or MDR TB 7 . In the first recognised outbreak of XDR-TB, 53 patients in KwaZulu-Natal, South Africa, who were co-infected with XDR TB and human immunodeficiency virus (HIV), survived for an average of 16 days, with a mortality of $98 \%{ }^{8}$ .XDR-TB raises concerns of a future tuberculosis epidemic with restricted treatment options, and jeopardises the major gains made in tuberculosis control.

For control of the spread of tuberculosis early diagnosis and treatment with suitable antitubercular drugs is necessary. The susceptibility of $M$. tuberculosis to a specific drug is typically determined by growing the bacteria in or on media containing that drug. Commonly the agar (LJ culture) and liquid culture are used for susceptibility testing of $M$. tuberculosis ${ }^{9}$. Because of the slow growth of the bacteria and the requirement for isolation before drug susceptibility testing, the agar proportion method usually requires 6-8 weeks to achieve results whereas the liquid culture method requires 12 days $^{10}$.

In this study sula liquid media has been used for isolation and drug susceptibility testing for first and second line anti- tubercular drugs. The results of the sula liquid media was compared with conventional LJ media.

\section{Materials and methods:}

Procedure of sputum collection

One spot sputum sample was collected in a container. On the initial hospital visit, the patient was provided a clean, dry, wide-neck, leak-proof container and requested him or her to cough deeply to produce a sputum specimen (3-5 ml volume)

\section{Smear preparation and fixation}

Smear was made from yellow purulent portion of the sputum using a bamboo stick. A good smear was spread evenly, $3 \mathrm{~cm}$ by $2 \mathrm{~cm}$ in size in the middle part of the slide which was neither too thick nor too thin. The sputum was left for 15-30 minutes for air dry. The smear was fixed by passing the slide over the flame 35 times for 3-4 seconds each time.

\section{Ziehl-Neelsen staining}

The smear was covered with carbol fuchsin stain. The stain was heated until vapors just begin to rise and the heated stain was poured over the slide and left for 10 minutes.

Then it was washed with clean water. The smear was then covered with $20 \% \mathrm{H}_{2} \mathrm{SO}_{4}$ and left for 3 minutes. Again the slide was washed with clear water. The slide was covered with methylene blue $0.3 \%$ for 1 minute. Then again washed off with clear water.

The back of the slide was wiped and placed in a draining rack to air-dry.

The smear was examined by microscope by using 100X oil immersion objective for red, straight or slightly curved rods, occurring singly or in small groups.

Decontamination and concentration of the specimen

Sputum was taken on the centrifuge tube (at least $2 \mathrm{ml}$, not more than $5 \mathrm{ml}$ ), then sterile beads and equal volume of $4 \% \mathrm{NaOH}$ was added. The cap of the container was tightened and vortexed to digest.

Allowed to stand for 15 minutes at room temperature

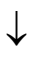

The tube was filled with phosphate buffer up to $50 \mathrm{ml}$ mark on the tube and vortexed

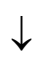

Centrifuged at $3000 \mathrm{rpm}$ for 20 minutes

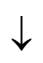

The supernatant portion was carefully poured off through a funnel in to a discard jar containing $5 \%$ phenol

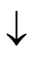

The deposit was used for culture 


\section{Method of conventional culture}

Two drops of deposit were inoculated on two slopes of Lowenstein-Jensen media. The media were incubated at $36^{\circ} \mathrm{C}$ after inoculation. Remaining deposit was kept at $-20^{\circ} \mathrm{C}$ for further short decontamination and inoculation if needed due to contamination. They were examined with in 3-5 days after inoculation for early recognition of rapidly growing Mycobacterium and of contaminated cultures, followed by once-weekly examination up to 8 weeks. Culture was reported as positive as soon as colonies of characteristics morphology constituted of acid-fast bacilli were recognized. The report of culture was done according to the number of colonies. The growth showing contaminations were excluded from the study.

\section{Conventional DST: Proportionate method}

\section{Preparation of bacterial suspension}

Colonies were scraped with a loop from all over the culture strain. A sterile, small thick walled screw-capped glass tube containing 5-7 clean glass beads was taken. The loop was gently shaken over the beads. 2 drops of sterile saline were added and frequently shaked.

The tube was allowed to stand for 15-30 minutes to settle the larger aggregates of bacteria. The homogenous upper part of the supernatant was transferred to another tube with similar dimension of the Mac Farland standard for the visual comparison with the standard. Using a sterile plastic Pasteur pipette, sterile saline was added until turbidity matched with McFarland no.1.

\section{Dilution and inoculation of bacterial suspension}

Serial dilution of the bacterial suspension was done in $10^{-1}, 10^{-2}, 10^{-3}, 10^{-4}$, and $10^{-5}$. Bacterial suspension was inoculated on a drug free medium which will act as growth control (GC). One tube was inoculated with suspension S3 (dilution $10^{-3}$ ) labeled as GC3 and another tube was inoculated with suspension S5 (dilution $10^{-5}$ ), labeled as GC5. Tubes containing the drug INH, RMP, SM and EMB were inoculated with S3 and S5.

\section{Proportion calculation:}

Number of colonies on

the drug media (GC5)

Number of colonies on

$\mathrm{X} 100=\%$

Proportion resistant

the control media (S5)

* Result is 1 or $>1$ is resistant. Result $<1$ is sensitive

Example: If INH in GC5 is 45 and S5 is 45 then

$\%$ proportion will be as follows

For INH $=\frac{45}{45} \begin{aligned} & \times 100=100 \% \text {, that is this } \\ & \text { stain is resistant to } \mathrm{INH}\end{aligned}$

\section{Slide DST:}

For slide DST, the method using Sula liquid medium with $10 \%$ locally collected goat serum was adopted described by Dissmann. In this media positive or negative growth as well as sensitivity pattern of first and second line drugs can be seen in the same settings. Eleven sputum smears were made on one end of longitudinally half autoclaved slides and then dried within safety cabinet and placed individually in sterile, heavy glass $28 \mathrm{ml}$ universal bottles containing $7 \mathrm{ml}$ medium. For each sample, two growth controls $(\mathrm{C} 1, \mathrm{C} 2)$, one half slides for RIF (1.0ig/ml), one for INH (1ig/ $\mathrm{ml})$, one for $\operatorname{EMB}(5.0 \mathrm{ig} / \mathrm{ml})$, one for $\mathrm{SM}=2 \mathrm{ug} /$ $\mathrm{ml}$, one for $\mathrm{K}(10 \mathrm{ig} / \mathrm{ml})$, one for $\mathrm{G}(8.0 \mathrm{ig} / \mathrm{ml})$, one for OF $(0.5 \grave{i g} / \mathrm{ml})$, one for PNB (P=500ig/ $/ \mathrm{ml})$ and one for nicotinamide $(\mathrm{N}=40 \mathrm{ig} / \mathrm{ml})$ were used. The bottles were incubated at $36^{\circ} \mathrm{C}$ for 12 days. After 12 days the bottles were removed from incubator and taken to a safety cabinet. After opening the bottle a forcep was used to remove the slide from the bottle, dipped the slides briefly in a universal container with rectified spirit and let them dried on a brown paper in the safety cabinet. After completion of the procedures, the spirit was discarding in a discard jar containing 5\% phenol. When the slides were dried, the smears were fixed in the flame of spirit lamp and arranged them in a non- sterile slide- rack placed in a staining jar and sufficient $1 \%$ carbolfuchsin was used to immerse the jar for 30 mins and then the other steps of Z-N staining was continued. ${ }^{11}$. 


\section{Results:}

Table I

Comparison of conventional and Slide drug susceptibility test (DST)

\begin{tabular}{|c|c|c|}
\hline Drugs & Conventional DST & Slide DST \\
\hline \multicolumn{3}{|l|}{$\overline{\mathrm{INH}}$} \\
\hline Sensitive & 49 & 49 \\
\hline Resistant & 51 & 51 \\
\hline \multicolumn{3}{|l|}{ RIF } \\
\hline Sensitive & 48 & 49 \\
\hline Resistant & 52 & 51 \\
\hline \multicolumn{3}{|l|}{ EMB } \\
\hline Sensitive & 59 & 61 \\
\hline Resistant & 41 & 39 \\
\hline \multicolumn{3}{|l|}{ SM } \\
\hline Sensitive & 60 & 57 \\
\hline Resistant & 40 & 43 \\
\hline \multicolumn{3}{|l|}{ G } \\
\hline Sensitive & - & 100 \\
\hline Resistant & - & 0 \\
\hline \multicolumn{3}{|l|}{ K } \\
\hline Sensitive & - & 99 \\
\hline Resistant & - & 1 \\
\hline \multicolumn{3}{|l|}{$\mathrm{OF}$} \\
\hline Sensitive & - & 90 \\
\hline Resistant & - & 10 \\
\hline \multicolumn{3}{|l|}{ NICO } \\
\hline Sensitive & - & 98 \\
\hline Resistant & - & 2 \\
\hline
\end{tabular}

Among the 100 tuberculosis cases, 51 were resistant to isoniazid, 52 to rifampicin, 41 to ethambutol and 40 to streptomycin by conventional drug susceptibility test (Table- I).

In Slide DST, among the 100 tuberculosis cases 51 were resistant to isoniazid, 51 to rifampicin, 39 to ethambutol and 43 to streptomycin, one to kanamycin and 10 to ofloxacin.

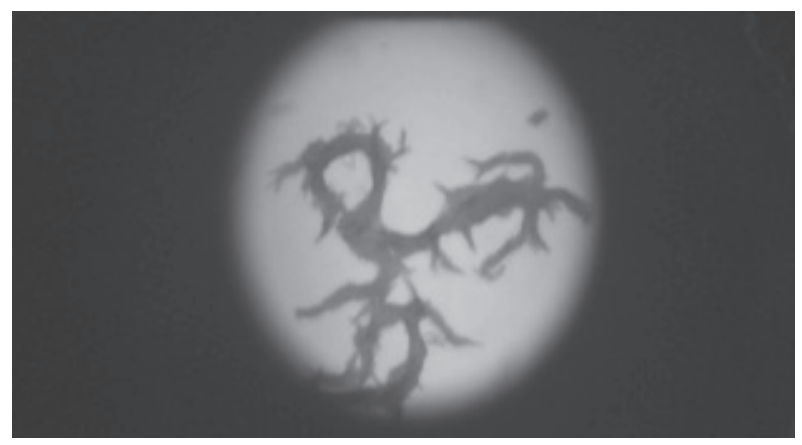

Fig.-1: Micro-colonies of M. tuberculosis

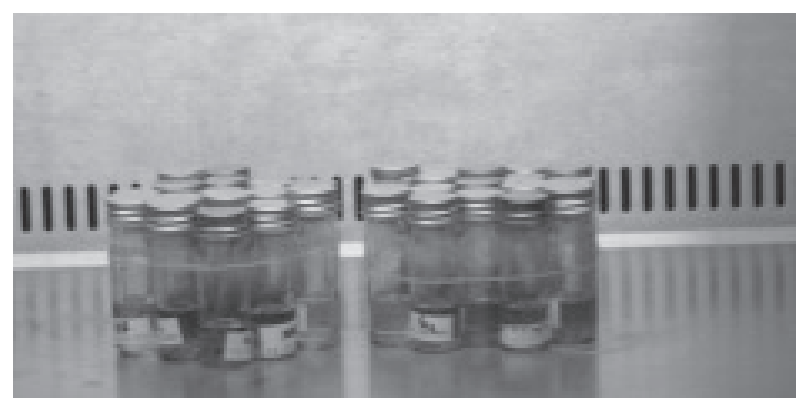

Fig.-2: sula liquid media

\section{Discussion:}

The early diagnosis and effective treatment of the open cases has been the focus of the tuberculosis control programmes all over the world. In this respect, direct smear examination by $\mathrm{ZN}$ staining has been the backbone of any tuberculosis control programme. But the drawback of the $\mathrm{ZN}$ smear examination is its low sensitivity. Conventional cultures on the LJ medium, though they are the gold standard, have the inherent disadvantage of time which is required to observe the growth. This may lead to a delay in the start of the treatment, facilitating the spread of the disease, or leading to the unnecessary treatment for the non pulmonary infections ${ }^{12}$.

Mycobacterium tuberculosis is an extremely slow growing organism. The possibility of improving on the standard culture media has been the attention of bacteriologist for a number of years. With this aim, comparative trials of various media have been carried out by a number of research workers. Pothmann (1949) obtained $20 \%$ more positive culture results with liquid media than with solid egg media. Kunzel (1950) found, on making 600 cultures on sula liquid media and on solid egg media, that on $50-60 \%$ more positive result could be obtained by sula liquid media than solid media. Saenz (1953) pointed out the advantages of liquid media: simplicity, rapidity and low contamination rate.

The sula liquid media is based on three principles: 1) Mycobacterium tuberculosis (MTB) grows faster in sula liquid media than on solid media 2) microscopic MTB growth can be detected earlier in liquid media than waiting for the macroscopic appearance of colonies on 
solid media, and that growth is characteristic of MTB, allowing it to be distinguished from atypical mycobacteria or fungal or bacterial contamination 3 ) the drugs isoniazid and rifampicin can be incorporated into the sula liquid media to allow for simultaneous direct detection of MDRTB, obviating the need for subculture to perform an indirect drug susceptibility test. ${ }^{16}$

The results of this study shows that, when the normal precautions are taken use of liquid media does not present any special problem such as high contamination. Using standardised drug susceptibility testing (DST) with conventional methods, 8 to 12 weeks are required to identify drug resistant tuberculosis on solid media (i.e., Lowenstein-Jensen medium). In general, these methods assess inhibition of $M$. tuberculosis growth in presence of antitubercular drugs to distinguish between susceptible and resistant strains. As the results usually take weeks, inappropriate choice of treatment regimen may result in death such as in case of XDR-TB especially in HIV co-infected patients. In addition, delayed diagnosis of drug resistance results in inadequate treatment, which may generate additional drug resistance and continued transmission in community. In liquid media the test sensitivity is better than traditional methods using $\mathrm{LJ}$ media with a turnaround time of 7 days for culture and drug sensitivity. Besides, it is cheap, simple and fairly accurate.

\section{Conclusion:}

The sula liquid media was found to be as sensitive as the $\mathrm{LJ}$ medium for the diagnosis of tuberculosis. The seven day turn around period of liquid media was a definite advantage over the $\mathrm{LJ}$ medium culture. The rapid culture can be used as an adjunct to the direct smear examination, especially in patients who are suspected of pulmonary tuberculosis, but remain sputum smear negative by direct microscopy.

\section{References:}

1. Jureen P, Enstrand L, Eriksson S, Alderborn A, Krabbe M, Hoffner SE. Rapid detection of Rifampicin resistance in Mycobacterium tuberculosis by using the pyrosequencing technique. J Clin Microbiol 2006; 44: 1925-29.
2. World Health Organization [Internet]. AntiTuberculosis drug resistance in the World. Report No. 4: The WHO / IUATLD Global Project on Antituberculosis Drug Resistance Surveillance. C2008. http://www.who.int/tb/publications / 2008/drs_rep ort4_26feb08.pdf. Accessed on June 10, 2012.

3. Paramasivan CN, Venkataraman P. Drug resistance in tuberculosis in India. Indian $\mathrm{J}$ Med Res 2004;120:377-86.

4. Chauhan LS. Challenge towards up scaling MDR TB under RNTCP. www.mohfw.nic.in/nrhm/ presentations / orrisa_workshop/3rd_day / ndcp_Isc4.pps

5. World Health Organization [Internet]. Countries that had reported at least one XDR-TB case by end March 2009; c2009. http://www.who.int/ tb/challenges/xdr/xdr_map_mar09.pdf. Accessed on June 10, 2012.

6. Sharma SK, George N, Kadhiravan T, Saha PK, Mishra HK, Hanif M. Prevalence of extensively drug-resistant tuberculosis among patients with multidrug-resistant tuberculosis: a retrospective hospital-based study. Indian $\mathrm{J}$ Med Res 2009; 130:392-5.

7. Migliori GB, Besozzi G, Girardi E, Kliiman K, Lange $\mathrm{C}$, Toungoussova OS, et al. Clinical and operational value of the extensively drug- resistant tuberculosis definition. Eur Respir J 2007;30:623-6.

8. Gandhi NR, Moll A, Sturm AW, Pawinski R, Govender T, Lalloo U, et al. Extensively drugresistant tuberculosis as a cause of death in patients co-infected with tuberculosis and HIV in a rural area of South Africa. Lancet 2006;368:1575-80.

9. Lin SYG, Lin SY, Probert W, Lo M, Desmond E. Rapid detection of isoniazid and rifampicin resistant mutations in Mycobacterium tuberculosis complex from cultures or smear positive sputa by use of molecular beacons. J Clin Microbiol 2004; 42: 4204-8.

10. Johnson R, Streicher EM, Louw GE, Warren RM, Van Helder PD, Victor TC. Drug resistance in Mycobacterium tuberculosis. Curr Issues Mol Biol. 2006;8(2):97-111.

11. Hamid A, Aung KJM, Hossain MA, Van Deun A. Early and rapid microscopy based diagnosis of true treatment failure and MDR-TB. Int J Tubercle Lung Dis 2006: 10; 1248-1254.

12. Jena J, Neema SK, Panda BN, Rajan KE. Comparative efficacy of the rapid slide culture of M.Tuberculosis and the conventional LJ medium culture in the diagnosis and management of pulmonary tuberculosis cases. Ind J Tub 1995; 42:151-54.

13. Pothmann F. J., 1949. Zbl. Bakt., I, Abt., Orig., 154, 152

14. Kunzel K. E., 1950. Zbl. Bakt., I, Abt., Orig., 156, 235

15. Saenz A., 1953. Presse med. 71, 381.

16. Brady MF, Coronel J, Gilman RH, Moore DA. The MODS method for diagnosis of tuberculosis and multidrug resistant tuberculosis. J Vis Exp 2008 ; Aug 11; pii: 845. doi: 10.3791/845. 